\title{
Intermingled cAMP, cGMP and calcium spatiotemporal dynamics in developing neuronal circuits
}

\author{
Stefania Averaimo $0^{1,2,3}$ and Xavier Nicol ${ }^{1,2,3}$ * \\ 1 UMR_7210, Centre National de la Recherche Scientifique, Paris, France \\ ${ }^{2}$ UMR S 968, Institut de la Vision, Sorbonne Universités, UPMC Univ Paris 06, Paris, France \\ ${ }^{3}$ U968, Institut National de la Santé et de la Recherche Médicale, Paris, France
}

Edited by:

Nicholas C. Spitzer, University of

California, San Diego, USA

\section{Reviewed by:}

Claudia Lodovichi, Venetian Institute of Molecular Medicine, Italy

Alberto Pereda, Albert Einstein

College of Medicine, USA

\section{*Correspondence:}

Xavier Nicol, UMR_S 968, Institut de la Vision, Sorbonne Universités, UPMC Univ Paris 06, 17 Rue Moreau, Paris F-75012, France e-mail: xavier.nicol@inserm.fr
cAMP critically modulates the development of neuronal connectivity. It is involved in a wide range of cellular processes that require independent regulation. However, our understanding of how this single second messenger achieves specific modulation of the signaling pathways involved remains incomplete. The subcellular compartmentalization and temporal regulation of cAMP signals have recently been identified as important coding strategies leading to specificity. Dynamic interactions of this cyclic nucleotide with other second messenger including calcium and cGMP are critically involved in the regulation of spatiotemporal control of CAMP. Recent technical improvements of fluorescent sensors facilitate cAMP monitoring, whereas optogenetic tools permit spatial and temporal control of cAMP manipulations, all of which enabled the direct investigation of spatiotemporal characteristics of CAMP modulation in developing neurons. Focusing on neuronal polarization, neurotransmitter specification, axon guidance, and refinement of neuronal connectivity, we summarize herein the recent advances in understanding the features of cAMP signals and their dynamic interactions with calcium and cGMP involved in shaping the nervous system.

Keywords: cAMP, cGMP, calcium, subcellular compartmentalization, kinetics, axon outgrowth, axon guidance, topographic maps

\section{INTRODUCTION}

The development of nervous system connectivity is a multistage process that requires neuron specification and polarization, axon guidance and targeting, as well as refinement of synaptic connections. All stages require second messenger cascades involving cAMP. This cyclic nucleotide is required for the control of transcription (Pham et al., 2001; Root et al., 2008), involved in polarizing immature neurons (Shelly et al., 2010), promotes axon outgrowth (Roisen et al., 1972; Cai et al., 2001; Shewan et al., 2002; Corredor et al., 2012), and regulates the response of growing axons to guidance molecules (Song et al., 1997; Höpker et al., 1999; Nishiyama et al., 2003; Nicol et al., 2006b). Later during development, cAMP is crucial for the refinement of neuronal connectivity and the precise choice of synaptic partners (Welker et al., 1996; Ravary et al., 2003). It is also involved in a wide range of cellular processes that are not related to the development of neuronal connectivity including sugar and lipid metabolism. However, we still have a very partial understanding of how this second messenger can achieve specificity for each of its downstream pathways.

Although the influence of cAMP on the developing nervous system has been identified decades ago, the shape, duration or subcellular localization of its variations in living neurons is still elusive. The spatiotemporal attributes of cAMP signals might provide the bases of a code explaining the specificity of a given cAMP modulation for its proper downstream pathway. cAMP signaling is tightly related to two other second messengers, cGMP and calcium, and their intricate regulation contributes to enlarge the coding strategies leading to the activation of unique signaling pathways (Borodinsky and Spitzer, 2006; Zaccolo and Movsesian, 2007). A recent and expanding set of tools now enables a precise study of the features of cAMP signals. This includes a growing number of genetically-encoded FRET-based biosensors that have been improved over time (Zhang et al., 2001; Nikolaev et al., 2004; Ponsioen et al., 2004; Polito et al., 2013) and allow the monitoring of cAMP signals in living neurons. This toolbox is complemented with recently implemented optogenetic adenylyl cyclases (ACs-the cAMP-synthesizing enzyme), providing full spatio-temporal control of cAMP concentration in living neurons (Schröder-Lang et al., 2007; Ryu et al., 2010; Hong et al., 2011; Stierl et al., 2011). Said utilities have recently been applied to the study of nervous system connectivity, revealing some of the cAMP coding strategies in developing neurons.

\section{MECHANISMS UNDERLYING THE GENERATION AND CONTROL OF SPATIOTEMPORAL CAMP SIGNALS}

The biological significance of cAMP signals during different steps of neuronal development relies on a tight control of both, their temporal and spatial features. Synthesis of cAMP is dependent on ACs, whereas the hydrolysis of cyclic nucleotides and 


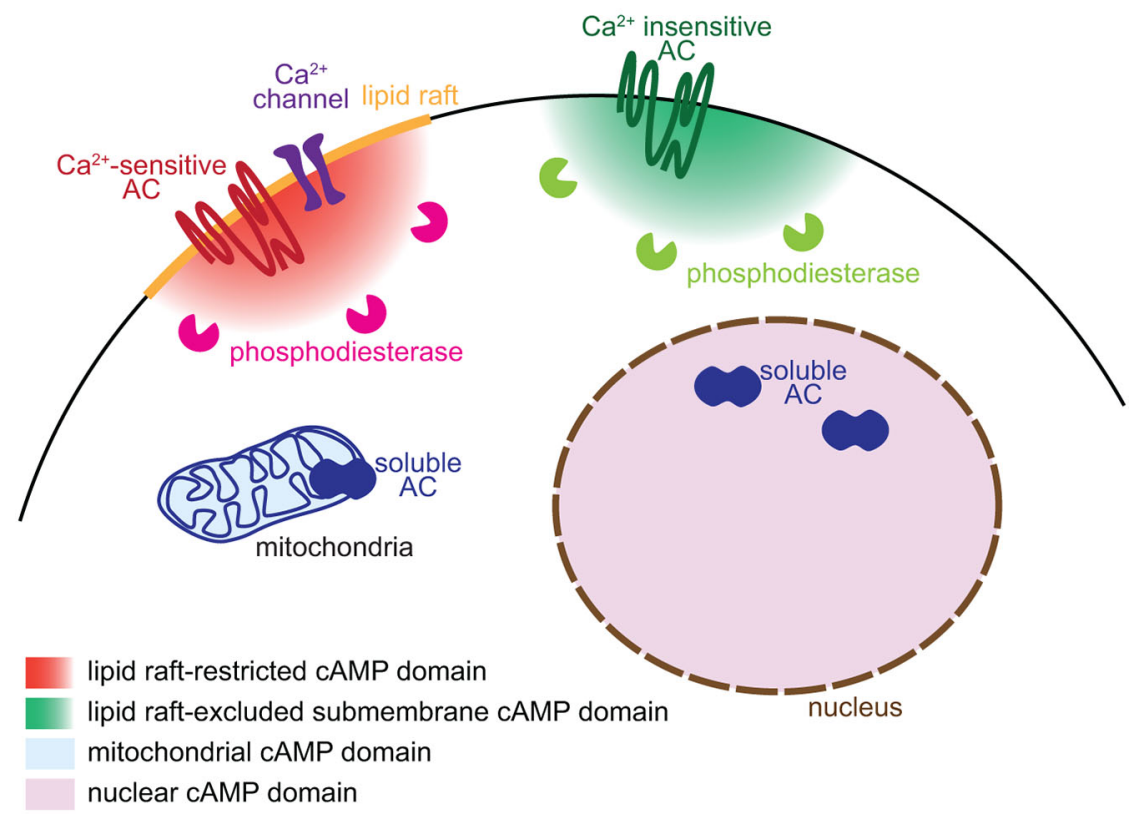

FIGURE 1 | Mechanisms underlying the generation of subcellular domains of cAMP. Distinct subcellular localizations of adenylyl cyclases and phosphodiesterases enable the generation of subcellular domains of CAMP. $\mathrm{Ca}^{2+}$-sensitive transmembrane ACs and their regulating $\mathrm{Ca}^{2+}$ channels are restricted to lipid rafts whereas the
$\mathrm{Ca}^{2+}$-independent $\mathrm{ACs}$ are excluded from this plasma membrane territory. Phosphodiesterases degrade cAMP close to its site of synthesis and limit the diffusion of the signal. The soluble AC is responsible of cAMP synthesis in discrete locations of the cell including the mitochondria and the nucleus. extinction of the signal relies on phosphodiesterases (PDEs). The diversity of regulation and localization of ACs (10 isoforms) and PDEs (more than 40 isoforms) offers a wide range of combination to shape specific signals in response to distinct stimuli (Omori and Kotera, 2007; Willoughby and Cooper, 2007; Kleppisch, 2009). The cooperation between ACs and PDEs is crucial to control the time of onset of cAMP elevation and to limit the spatial and temporal expansion of the signal, forming the relevant coding strategies controlling specific activation of cAMP downstream effectors (Figure 1).

Different AC isoforms are targeted to distinct cellular microdomains, providing the basis of locally generated cAMP signals. sAC/AC10 is associated with intracellular compartments including mitochondria, centrosome and nucleus (Zippin et al., 2003; Valsecchi et al., 2014). The calcium-sensitive transmembrane ACs (AC1, 3, 5, 6 and 8) are tethered to lipid rafts, a cholesterol- and sphyngolipid-enriched compartment of the plasma membrane. In contrast, the calcium-independent ACs (AC2, 4, 7 and 9) are targeted to the plasma membrane but excluded from lipid rafts (Willoughby and Cooper, 2007; Figure 1). Targeting or exclusion of specific AC isoforms from lipid rafts might be the crucial molecular correlate of distinct cAMP concentrations and signaling in compartments of the plasma membrane (Depry et al., 2011). Lipid rafts compartmentalize cAMP signals induced by beta adrenergic receptors in cardiomyocytes (Agarwal et al., 2011). In platelets, compartmentalized cAMP signals are created by lipid rafts and the cytoskeleton (Raslan and Naseem, 2014). This plasma membrane compartment is of particular interest to understand the development of neuronal connectivity. Its integrity is crucial for BDNF-, netrin-1- and Sema3A-induced, but not glutamate-dependent turning of $X$. laevis axons (Guirland et al., 2004). Evaluating whether lipid raft-restricted cAMP signals are required for axon pathfinding presents a promising approach to deciphering the second-messenger codes involved in axon guidance. Precise localization of ACs is also crucial for the coordination between second messengers. Interaction between $\mathrm{AC} 8$ and the pore component of the store operated calcium channels ensures a dynamic and coordinated relation between cAMP and calcium (Willoughby et al., 2012).

The cAMP turnover, balanced by synthesis and degradation, requires a tight regulation. Like ACs, PDEs are crucial for the spatio-temporal control of cAMP signaling. Their distinct intracellular localization, kinetics and regulatory mechanisms enable to shape a wide range of signals that differ in their spatiotemporal features and upstream regulators. Compartmentalization of PDEs is responsible for simultaneously generating multiple and contiguous cAMP domains, even far from the site of synthesis (Terrin et al., 2006). According to this model, synthesized cAMP is free to diffuse. The 
specific activation of only a subset of its downstream pathways relies on the restricted subcellular localization of PDEs, acting locally as a cAMP sink to prevent the activation of downstream effectors (Figure 1). The cAMP-specific PDE4 family is crucial for this process in a wide number of cell types. In cardiomyocytes, the activation of PDE4D spatially limits the diffusion of cAMP, modulating cAMP-dependent protein kinase A (PKA) activation and the subsequent increase in calcium cycling required for contractile responses in the heart (Liu et al., 2012). In fibroblasts, PDE4B and PDE4D modulates cAMP concentration in discrete domains near the plasma membrane and are involved in distinct signaling pathways (Blackman et al., 2011). In neurons, the PDE4 family is involved in the functional compartmentation of cAMP, modulating the propagation of PKA activation from the plasma membrane to the nucleus (Castro et al., 2010; Vincent et al., 2012).

For ACs and PDEs to properly control the localization of cAMP signals, the targeting of these enzymes is tightly regulated. A-kinase anchoring proteins (AKAPs) are critical for this process. AKAP isoforms are targeted to distinct subcellular compartments and modulate the spatial spread of cAMP, binding at least some isoform of PDEs and ACs (Piggott et al., 2008; Willoughby et al., 2010; Delint-Ramirez et al., 2011; Terrin et al., 2012). In addition, AKAPs bind downstream effectors of cAMP including PKA and the cAMP-stimulated GDP exchange factors (Epacs), segregating distinct cAMP downstream pathways (Wong and Scott, 2004; McConnachie et al., 2006). Although to date there are only a few studies focusing on the spatial restriction of cAMP signals by AKAPs in developing neurons, these anchoring proteins have been extensively studied in other cell types. For instance, in airway smooth muscle cells, AKAPs modulates cAMP accumulation in response to $\beta 2$-adrenergic agonists. PKA activation in turn phosphorylates PDE4, increasing its activity and reducing cAMP concentration in specific domains where AKAP proteins are localized (Horvat et al., 2012). In the nucleus, AKAPs have been proposed to control a PKA/PDE modulated cAMP signal different from that in the cytosol microdomain (Sample et al., 2012). Indeed, cAMP signaling at the plasma membrane followed by slow diffusion into the nucleus results into slow kinetics of PKA activity likely to be limited by the translocation of the catalytic domain of PKA from the cytosol to the nucleus. Indeed, PDEs keep the cAMP concentration in the nucleus too low to activate PKA. However, a direct activation of cAMP synthesis in the nucleus would result into fast kinetics of the nuclear PKA response. In this case, the spatio-temporal modulation of cAMP is responsible for a kinetically distinct activation of PKA, and the local negative regulator PDE4 contributes to establishing a local signaling threshold to convert spatial second messenger signals to temporal control of kinase activity. Finally, a dynamic and delicate control of cAMP signals has also been identified in the centrosome, a key subcellular structure for migration and cell cycle progression. In this subcellular domain, cAMP concentration is independent on cAMP levels in the cytosol and relies on PDE4D3 and PKA anchoring to AKAP 450 (Terrin et al., 2012).

\section{EARLY EVENTS: NEURONAL POLARIZATION AND NEUROTRANSMITTER SPECIFICATION}

The polarization of postmitotic neurons leads to the segregation of two distinct subcellular compartments: a single axon and a somato-dendritic compartment including multiple dendrites (Arimura and Kaibuchi, 2007; Barnes et al., 2008). Axon/dendrite specification of undifferentiated neurites involves cyclic nucleotide signaling aiming at the development of a single axon in each neuron. The use of cAMP and cGMP reporters enabled the identification of local cyclic nucleotide signals regulating axon/dendrite specification. cAMP initiates a positive feedback involving phosphorylation of the serine/threonine kinase LKB1 (Liver Kinase B1) and its interactor STRAD (STE20-related adapter protein), leading to differentiation and stabilization of the axon (Shelly et al., 2007). In contrast, cGMP favors the differentiation of immature neurites into mature dentrites (Shelly et al., 2010). Local and distal interactions between these two second messengers create two subcellular compartments that define the polarity of the neuron. Local cAMP elevation at the tip of a neurite leads to a local reduction of cGMP concentration, and a distal cAMP suppression and cGMP elevation in the other neurites and vice versa (Shelly et al., 2010; Figure 2). This local modulation of cyclic nucleotide levels is observed in maturing neurons in response to extracellular cues that favor either axon or dendrite initiation like BDNF or Semaphorin3A respectively (Shelly et al., 2011), or by activation of $\mathrm{GABA}_{B}$ receptors (Bony et al., 2013). These observations emphasize the influence of local cyclic nucleotide signaling for axono- and dendritogenesis.

A second feature of neuronal differentiation is the specification of neurotransmitters. This is a crucial step in the development of the nervous system to ensure appropriate connectivity and to finely tune the balance between excitation and inhibition. In developing neurons, spontaneous calcium spikes play a critical role in the choice of neurotransmitter. The frequency of these calcium transients dictates the expression of an inhibitory or excitatory neurotransmitter. Expression of the hyperpolarizing potassium channel Kir2.1 suppresses calcium spikes in spinal $X$. laevis neurons and triggers the expression of the excitatory neurotransmitters machinery, including the glutamate vesicular transporter (VGluT) and choline acetyltransferase transporters (ChAT). In contrast, overexpression of the voltage gated sodium channel $\mathrm{Na}_{v} 1.2$ increases calcium spike frequency and the number of the inhibitory GABAergic and glycinergic spinal neurons at the expense of VGluT and ChAT-expressing neurons, demonstrating that pattern calcium activity affects neuronal differentiation (Borodinsky et al., 2004). Metabotropic GABA receptors and group III metabotropic glutamate receptors regulate PKA activity, one of the main cAMP effectors, and modulate the generation of calcium spikes (Root et al., 2008). Interestingly, cAMP transients, rather than sustained elevation of this second messenger, are generated in X. laevis spinal neurons at the same developmental stage than calcium spikes that regulate neurotransmitter specification (Gorbunova and Spitzer, 2002). cAMP transients and calcium spikes are interdependent. Bursts of calcium spikes induce slow cAMP transients (3-7 $\mathrm{min}$ in young and older neurons respectively) and increasing cAMP 


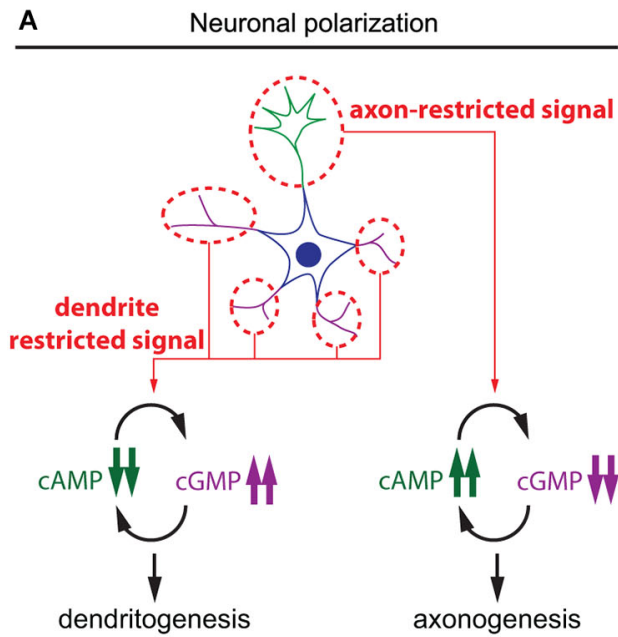

FIGURE 2 | Spatiotemporal dynamics of cAMP during neuronal polarization and neurotransmitter specification. (A) Local interactions between CAMP and CGMP determine neurite maturation into axon or dendrite. High cAMP and low cGMP concentrations promote axonogenesis, whereas low cAMP and high cGMP lead to

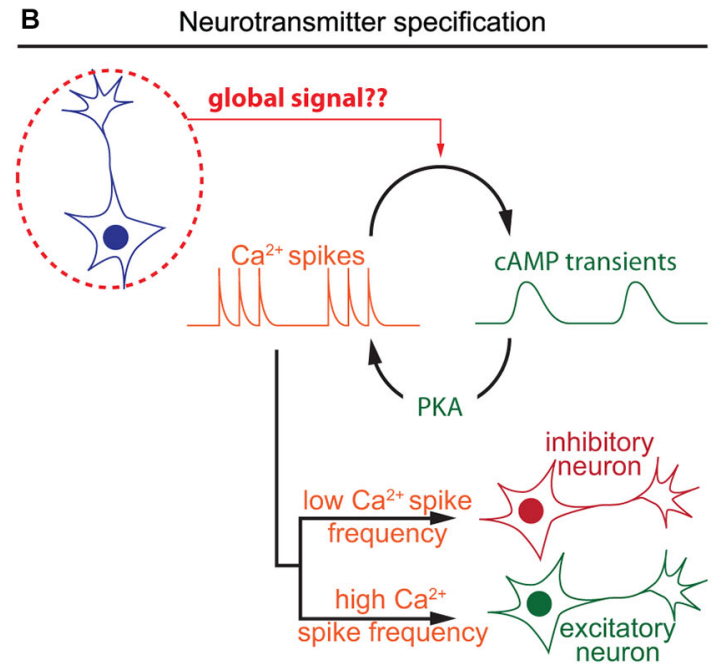

dentritogenesis. (B) In developing neurons, cAMP transients and calcium spikes are interdependent. The choice of expression of excitatory or an inhibitory neurotransmitter is modulated by the frequency of calcium spikes, which is regulated by the activity of a set of kinases including the cAMP effector PKA. concentration concomitantly increases the frequency of calcium spikes (Gorbunova and Spitzer, 2002; Figure 2). This reciprocal interaction between calcium and cAMP signal dynamics has been observed in a wide range of non-neuronal cell types (Landa et al., 2005; Borodinsky and Spitzer, 2006; Dyachok et al., 2006; Willoughby and Cooper, 2006). However, a possible direct relationship between cAMP transients and neurotransmitter specification has not been investigated. It would be of interest to evaluate if the frequency of cAMP transients influences neurotransmitter specification in developing neurons, like the frequency of calcium spikes.

\section{AXON OUTGROWTH}

Once neurons are polarized, axons grow over long distances to reach their synaptic partners. Axon elongation is dependent on cAMP signaling and high concentration of this second messenger favors axon outgrowth (Roisen et al., 1972; Cai et al., 2001; Shewan et al., 2002; Corredor et al., 2012). In this process as well, cAMP signaling is tightly linked to calcium to regulate axon outgrowth. Transient calcium elevations, termed calcium waves, have been visualized in the growth cone of extending axons. Calcium waves cover the entire growth cone at once and their kinetics is relatively slow. The frequency is inversely proportional to the speed of axon outgrowth in a wide range of models: lower frequency of calcium transients correlates with a high rate of axonal growth, while a high frequency is observed in slow growing axons (Gomez et al., 1995; Gomez and Spitzer, 1999; Tang et al., 2003). For instance in the developing spinal cord of X. laevis, Rohon-Beard neurons, ventral motor neurons and ascending interneurons exhibit a low frequency of calcium transients and a rapid outgrowth. In contrast, the frequency of calcium transients is higher in the slow growing dorso-lateral ascending interneurons (Gomez and Spitzer, 1999). A direct link between cAMP signals modulating axon outgrowth and calcium waves in growth cones has not been investigated so far. However, cAMP transients have been detected in axonal growth cones exposed to netrin-1, a guidance molecule that modulates both, the direction and the speed of axon elongation. cAMP transients require extracellular calcium influx and coincide with an increased frequency of calcium waves, that are not dependent on transmembrane AC activity (Nicol et al., 2011; Figure 3). The transient change in CAMP suggests that a temporal control might contribute to the coding strategy of cAMP signals regulating axon outgrowth.

Only cAMP signals from a subset of ACs are able to modulate axon outgrowth. The synthesis of cAMP by soluble AC (sAC/AC10) but not transmembrane ACs (AC1 to 9) is required for the intrinsic ability of axons to grow (Corredor et al., 2012). sAC/AC10 is also critical for netrin-1-induced outgrowth (Wu et al., 2006), but does not contribute to the cAMP-dependent modulation of axon pathfinding by netrin-1 (Moore et al., 2008). This suggests that cAMP signals generated by different ACs are not identical, although the features allowing the distinction between diverse cAMP signals have not been identified. A likely hypothesis is that subcellular localization of signals would provide a code to link each cAMP signal to its appropriate downstream pathway. Indeed $\mathrm{sAC/AC10}$ is restricted to intracellular compartments in non-neuronal cells (Zippin et al., 2003; Feng et al., 2006), whereas the other ACs are transmembrane proteins.

\section{AXON GUIDANCE}

To reach their synaptic partners, axons follow stereotypic paths, guided by attractive and repulsive cues, which they encounter 


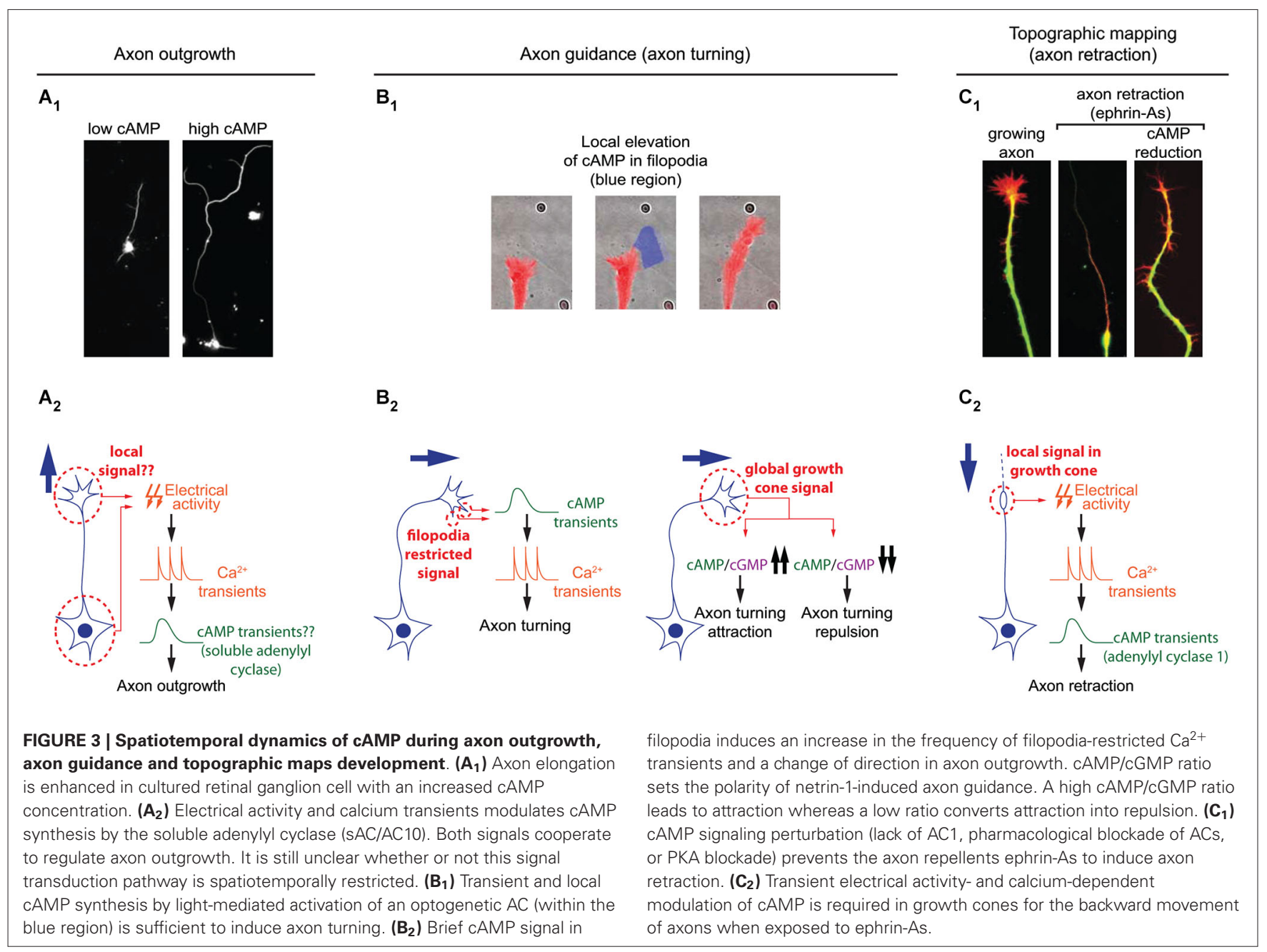

on the way to their final targets. cAMP signaling is a crucial modulator of axonal response to guidance molecules and enables the conversion of attractive cues into repulsive ones and vice versa (Ming et al., 1997; Song et al., 1997; Höpker et al., 1999; Murray et al., 2009). However, the spatio-temporal dynamics of cAMP signals regulating axon guidance have been investigated only recently. As during neurotransmitter specification and axon outgrowth, cAMP signaling involved in axon pathfinding is linked to calcium. Calcium signaling is critical for axonal response to guidance molecules (Hong et al., 2000; Nishiyama et al., 2003; Li et al., 2009). In addition to slow calcium waves regulating axon outgrowth, fast calcium transients restricted to filopodia have been visualized in developing axons (Gomez et al., 2001). An asymmetric frequency of calcium transients in filopodia from both sides of the growth cone is able to orient axon outgrowth (Gomez et al., 2001; Robles et al., 2003). Guidance molecules such as netrin-1 modulate the frequency of filopodial calcium transients. Transient elevations of cAMP precede and are required for the increase in frequency of local filopodial calcium transients (Nicol et al., 2011), making cAMP crucial to orient netrin-1induced axon outgrowth. Although netrin-1 exposure also drives an increase in cAMP concentration in the central domain of the growth cone, this signal is not involved in the regulation of axon growth direction (Nicol et al., 2011). This suggests that axon guidance molecules can induce cAMP synthesis in a restricted area of the growth cone for a limited duration. This idea is supported by observations of axon turning generated by exogenous spatially- and temporally-restricted cAMP signals in vitro (Munck et al., 2004; Nicol et al., 2011). In vivo, transient cAMP elevations are sufficient to restore outgrowth of commissural axons towards the ventral midline of the spinal cord when DCC-dependent netrin-1 signaling is blocked. In contrast, sustained increase or decrease of cAMP concentration leads to abnormal trajectories of spinal commissural axons in X. laevis (Nicol et al., 2011), suggesting that spatio-temporal features of cAMP signals are crucial for the modulation of axonal pathfinding (Figure 3).

The regulation of axon pathfinding by cAMP signaling tightly controlled in space and time is most likely not the only cAMPdependent process regulating axon guidance. Axon turning can be modulated by sustained and global manipulations of PKA activity that are sufficient to modify the amplitude of imposed fast and local calcium transients dependent on ryanodine receptors (Ooashi et al., 2005). The resting cAMP concentration is tuned 
by adhesion molecules such as laminin that also regulate axon guidance (Höpker et al., 1999; Ooashi et al., 2005). In addition, cAMP interacts with cGMP signaling, often counteracting its effects. Whereas high cAMP concentration favors attraction, cGMP is associated with axonal repulsion. A high cAMP/cGMP ratio in spinal axons leads to attraction by netrin-1, whereas a low ratio converts this attraction into repulsion. This highlights the relevance of relative cAMP levels compared to cGMP rather than an absolute concentration of one or the other cyclic nucleotide (Nishiyama et al., 2003; Figure 3). The antagonizing effect of cAMP and cGMP might be due to their opposite regulation of common downstream effectors. Both second messengers modulate the amplitude of the ryanodine receptordependent local calcium transient. The cAMP/PKA pathway promotes ryanodine receptor-mediated calcium induced calcium release, whereas cGMP and its downstream effector protein kinase $\mathrm{G}$ (PKG) reduces the ryanodine receptor-dependent mobilization of internal calcium stores (Ooashi et al., 2005; Tojima et al., 2009). Simultaneous imaging of cAMP and cGMP in growing axons emphasized a temporally correlated regulation of cAMP and cGMP in growth cones. As during axonogenesis, an increase of cAMP correlates with a reduction in cGMP concentration (Kobayashi et al., 2013). The mechanisms underlying these opposite modulations are unclear. In other systems, inverse regulation of both cyclic nucleotides are related to PDE activity (Shelly et al., 2010; Polito et al., 2013). So far, not much attention has been attributed to evaluating the impact of these cAMP/cGMP degrading enzymes, which might be crucial players in cyclic nucleotide oscillations in axon guidance.

\section{AXON BRANCHING AND TARGETING (TOPOGRAPHIC MAPS)}

Once axons have reached their targets, dense terminal axonal arbors develop. The precise positioning of this termination zone is tightly controlled and crucial for appropriate wiring of the nervous system. This process has been extensively studied in sensory systems, in which projections are topographically organized. Axonal arbors are organized in their target regions in correlation with the information they carry. For instance, axons originating from neighboring retinal ganglion cells arborize in neighboring areas in their targets. Similarly, olfactory neurons expressing the same olfactory receptor target the same region of the olfactory bulb. cAMP signaling is crucial for the development of topographic maps. Among the ACs tested, only two are required for topographic map formation: $\mathrm{AC1}$ in the visual and somatosensory systems (Welker et al., 1996; Ravary et al., 2003; Nicol et al., 2006a), and AC3 in the olfactory projections (Wong et al., 2000; Col et al., 2007; Zou et al., 2007). In AC1 and AC3 knock-out mice, ectopic axonal branches fail to be pruned during development resulting in larger termination zones. In contrast, $\mathrm{AC} 1$ and $\mathrm{AC} 3$ are not involved in axon outgrowth, a process requiring sAC/AC10 (Wu et al., 2006; Corredor et al., 2012). The distinct roles of each $\mathrm{AC}$ suggest that they generate cAMP signals with unique features.

AC1-dependent cAMP signaling involved in the development of topographic maps requires calcium. Retinal axons lacking this calcium-activated AC (Fagan et al., 2000) or exposed to calcium-free extracellular medium show the same phenotype when exposed to the axon repellent ephrin-As: in both conditions axons fail to retract (Nicol et al., 2006b, 2007). This reduced retraction is likely to contribute to the impaired axonal pruning seen in $\mathrm{AC}^{-/-}$mice since ephrin-As are crucial for the development of retinal topography (Frisén et al., 1998; Feldheim et al., 2000). Ephrin-A-induced retraction also requires electrical activity. In absence of electrical activity, short and repeated cAMP pulses in growth cones are sufficient to rescue axonal retraction. In contrast, global and sustained elevation of cAMP does not rescue axon retraction. Sustained high or low cAMP concentration is sufficient to prevent ephrin-A-induced repulsion, suggesting that the temporal features of cAMP signaling is crucial for axonal retraction and the development of topographic maps (Nicol et al., 2007; Figure 3).

AC3 is required for the correct antero-posterior position in terminal zones of olfactory projections and for the convergence of olfactory axons expressing the same olfactory receptor into the same glomerulus (Wong et al., 2000; Col et al., 2007; Zou et al., 2007). The cAMP-dependent signaling pathways involved differ in these two developmental processes, only one of them interacting with calcium signaling. AC3-dependent cAMP signaling regulates the expression of the axon guidance molecule semaphorin-3A and its receptor neuropilin-1 to modulate the position of axonal arbors along the antero-posterior axis in a CREB-dependent mechanism (Imai et al., 2006). In contrast, $\mathrm{AC} 3$-dependent activation of the cyclic nucleotide gated calcium channel $\alpha 2$ is required for the development of glomeruli receiving axons expressing the same olfactory receptor (Serizawa et al., 2006). The spatiotemporal analysis of cAMP modulation in developing olfactory neurons revealed that activation of olfactory receptors at axonal growth cones induces a local increase of cAMP and calcium, followed by the nuclear translocation of the catalytic subunit of PKA (Maritan et al., 2009). Exposure of olfactory axon terminals to odorants also leads to a local cAMP- and calcium-dependent increase of cGMP (Pietrobon et al., 2011). Activation of downstream cGMP signaling is sufficient but not necessary to induce CREB phosphorylation in the nucleus of olfactory neurons (Pietrobon et al., 2011), suggesting that cGMP might be involved in the modulation of gene expression during olfactory map development.

\section{ACTIVITY-DEPENDENT AXONAL AND SYNAPTIC COMPETITION}

Once axonal branches are positioned in their targets, they carefully choose their synaptic partners, often through an axonal or synaptic competition process. cAMP has a crucial role in modulating this competition that represents a critical process for the development of a properly organized neuronal connectivity (Luo and O'Leary, 2005; Luo and Flanagan, 2007). Competition is dependent on electrical activity, and its regulation by cAMP often relates to spontaneous activity generated during development (Penn et al., 1998; Stellwagen and Shatz, 2002; Zhang et al., 2012; Furman et al., 2013). In the developing retina, spontaneous calcium waves appear prior to vision. The frequency and propagation of these calcium waves are modulated by cAMP, with a higher 


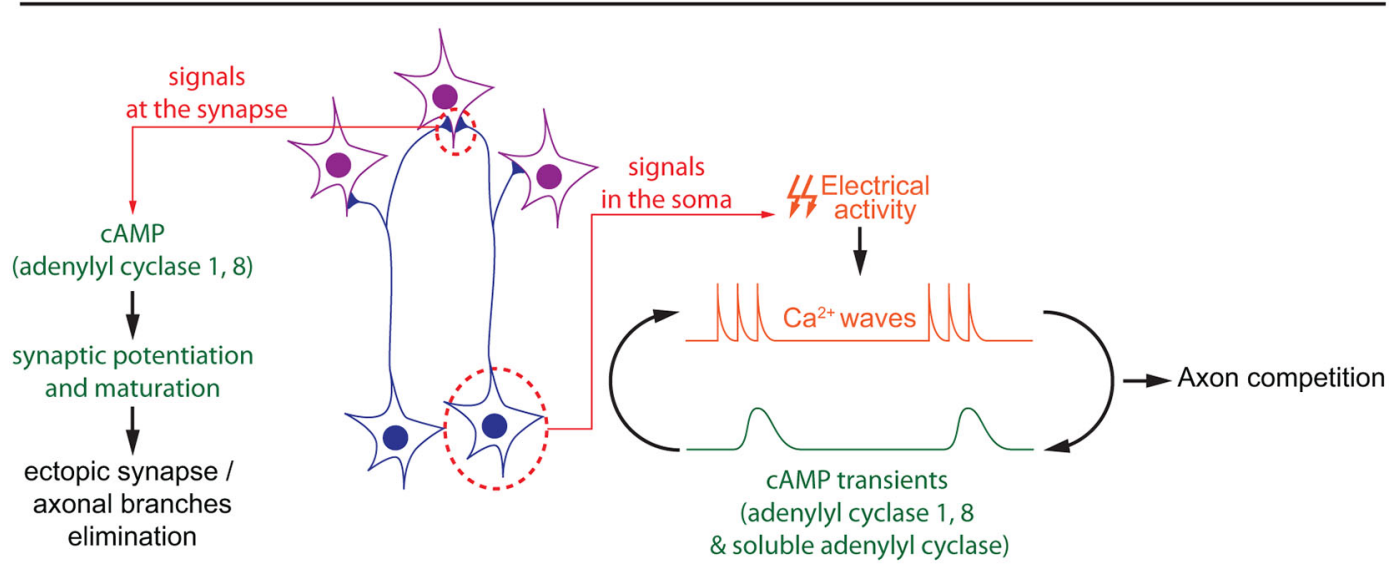

FIGURE 4 | Spatiotemporal dynamics of cAMP during developmental pruning of ectopic axonal branches, and synaptic competition. CAMP is required in two subcellular compartments during the synaptic refinement of neuronal projections. In the soma of retinal ganglion cells, cAMP oscillations and spontaneous calcium waves are interdependent. Spontaneous calcium waves are required for competition between axons in their targets. In the synapses, CAMP signaling mediated by adenylyl cyclases 1 and 8 is involved in synaptic potentiation and hebbian mechanisms, which are crucial to eliminate misplaced synapses. frequency and a propagation over longer distances when cAMP is increased (Stellwagen et al., 1999; Stellwagen and Shatz, 2002). Interactions between cAMP and calcium waves in the developing retina are bidirectional: depolarization-induced calcium influx is sufficient to produce brief cAMP elevation, and cAMP transients are spontaneously generated in post-natal retinal ganglion cells (Dunn et al., 2006; Figure 4). Like the cAMP elevations observed in X. laevis spinal neurons, spontaneous cAMP / PKA activity transients in the developing retina are observed upon long but not short bursts of electrical activity (Gorbunova and Spitzer, 2002; Dunn et al., 2006). Surprisingly, the genetic removal of AC1, the main calcium-stimulated $\mathrm{AC}$ expressed in retinal ganglion cells, is not sufficient to perturb depolarization-induced cAMP elevations. In AC1/AC8 double knock-out mice, cAMP transients only exhibit a reduced amplitude and are abolished only with pharmacological blockade of both, transmembrane (AC1 to 9) and soluble (sAC/AC10) ACs, suggesting that several ACs cooperate to generate brief cAMP / PKA activity transients in the retina (Dunn et al., 2009).

During development, cAMP-dependent spontaneous electrical activity in the retina is crucial for the precise organization of retinal axons in their targets. Retinal axons from each eye arborize in non-overlapping regions of their targets in a competition-dependent mechanism. When retinal calcium waves are perturbed, the normally well segregated territories occupied by axons from each eye overlap (Rossi et al., 2001; Muir-Robinson et al., 2002). Although AC1 is not required for the generation of cAMP transients or calcium waves in the developing retina (Dunn et al., 2009; Dhande et al., 2012), its genetic removal prevents the segregation of retinal projections in eye-specific territories in the dorso-lateral geniculate nucleus and the superior colliculus (Ravary et al., 2003; Nicol et al., 2006a), revealing that other cAMP signals are dependent on this particular AC. The stimulation of AC1 by calcium influx (Fagan et al., 2000), and the phenotypic similarity between $\mathrm{AC}^{-/-}$mice and models displaying altered spontaneous retinal waves or impaired electrical activity suggests that AC1 and calcium waves are part of the same signaling pathway regulating the development of eye-specific territories (Rossi et al., 2001; Muir-Robinson et al., 2002; Dhande et al., 2011). Converging evidence indicates that the deficit in the segregation of eye-specific territories in $\mathrm{AC}^{-/-}$mice is dependent on AC activity in retinal ganglion cells but not their targets (Nicol et al., 2006b; Dhande et al., 2012). Since cAMP signaling is not affected in the soma of retinal ganglion cells in AC1deficient mice, it is probable that cAMP signaling restricted to the axonal/synaptic compartment plays a role in the competition leading to segregation of eye-specific territories. AC1 is required for synaptic maturation and potentiation, a process required for the refinement of retinal connections (Lu et al., 2006; Iwasato et al., 2008; Figure 4). However, the features of cAMP signaling required for synaptic competition need to be characterized in more detail to improve our understanding of the signaling code involved in synaptic competition.

\section{CONCLUSION}

cAMP is involved in a wide range of processes influencing the development of nervous system connectivity. We summarize the recent progress in investigating the specific modulation of these multiple processes by spatio-temporally distinct cAMP signals. Temporal control of cAMP signals in subcellular compartments is a potential coding strategy to translate distinct cAMP signals into activation of specific downstream pathways in developing neurons. Crosstalk with other second messenger systems (cGMP and calcium) are of special importance to gain new insight into the spatiotemporal control of cAMP signals. Pursuing the analysis of these coding strategies will provide a better understanding of the regulatory second messenger networks shaping neuronal connectivity. 


\section{ACKNOWLEDGMENTS}

We thank Heike Blockus for helpful critical reading of the manuscript. The research in our laboratory is supported by Fondation de France, and by the French National Agency for Research (ANR) in the frame of the PDOC program (ANR-11-PDOC-0004), and of the LABEX LIFESENSES (ANR10-LABX-65) within the Investissements d'Avenir program (ANR-11-IDEX-0004-02).

\section{REFERENCES}

Agarwal, S. R., MacDougall, D. A., Tyser, R., Pugh, S. D., Calaghan, S. C., and Harvey, R. D. (2011). Effects of cholesterol depletion on compartmentalized cAMP responses in adult cardiac myocytes. J. Mol. Cell. Cardiol. 50, 500-509. doi: 10.1016/j.yjmcc.2010.11.015

Arimura, N., and Kaibuchi, K. (2007). Neuronal polarity: from extracellular signals to intracellular mechanisms. Nat. Rev. Neurosci. 8, 194-205. doi: 10. 1038/nrn2056

Barnes, A. P., Solecki, D., and Polleux, F. (2008). New insights into the molecular mechanisms specifying neuronal polarity in vivo. Curr. Opin. Neurobiol. 18, 4452. doi: 10.1016/j.conb.2008.05.003

Blackman, B. E., Horner, K., Heidmann, J., Wang, D., Richter, W., Rich, T. C., et al. (2011). PDE4D and PDE4B function in distinct subcellular compartments in mouse embryonic fibroblasts. J. Biol. Chem. 286, 12590-12601. doi: 10.1074/jbc. m110.203604

Bony, G., Szczurkowska, J., Tamagno, I., Shelly, M., Contestabile, A., and Cancedda, L. (2013). Non-hyperpolarizing GABAB receptor activation regulates neuronal migration and neurite growth and specification by cAMP/LKB1. Nat. Commun. 4:1800. doi: $10.1038 /$ ncomms 2820

Borodinsky, L. N., Root, C. M., Cronin, J. A., Sann, S. B., Gu, X., and Spitzer, N. C. (2004). Activity-dependent homeostatic specification of transmitter expression in embryonic neurons. Nature 429, 523-530. doi: 10.1038/nature02518

Borodinsky, L. N., and Spitzer, N. C. (2006). Second messenger pas de deux: the coordinated dance between calcium and cAMP. Sci. STKE 2006:pe22. doi: 10. 1126/stke.3362006pe22

Cai, D., Qiu, J., Cao, Z., McAtee, M., Bregman, B. S., and Filbin, M. T. (2001). Neuronal cyclic AMP controls the developmental loss in ability of axons to regenerate. J. Neurosci. 21, 4731-4739.

Castro, L. R. V., Gervasi, N., Guiot, E., Cavellini, L., Nikolaev, V. O., PaupardinTritsch, D., et al. (2010). Type 4 phosphodiesterase plays different integrating roles in different cellular domains in pyramidal cortical neurons. J. Neurosci. 30 , 6143-6151. doi: 10.1523/jneurosci.5851-09.2010

Col, J. A. D., Matsuo, T., Storm, D. R., and Rodriguez, I. (2007). Adenylyl cyclasedependent axonal targeting in the olfactory system. Development 134, 24812489. doi: 10.1242/dev.006346

Corredor, R. G., Trakhtenberg, E. F., Pita-Thomas, W., Jin, X., Hu, Y., and Goldberg, J. L. (2012). Soluble adenylyl cyclase activity is necessary for retinal ganglion cell survival and axon growth. J. Neurosci. 32, 7734-7744. doi: 10.1523/jneurosci. 5288-11.2012

Delint-Ramirez, I., Willoughby, D., Hammond, G. V. R., Ayling, L. J., and Cooper, D. M. F. (2011). Palmitoylation targets AKAP79 protein to lipid rafts and promotes its regulation of calcium-sensitive adenylyl cyclase type 8 . J. Biol. Chem. 286, 32962-32975. doi: 10.1074/jbc.m111.243899

Depry, C., Allen, M. D., and Zhang, J. (2011). Visualization of PKA activity in plasma membrane microdomains. Mol. Biosyst. 7, 52-58. doi: 10 1039/c0mb00079e

Dhande, O. S., Bhatt, S., Anishchenko, A., Elstrott, J., Iwasato, T., Swindell, E. C., et al. (2012). Role of adenylate cyclase 1 in retinofugal map development. $J$. Comp. Neurol. 520, 1562-1583. doi: 10.1002/cne.23000

Dhande, O. S., Hua, E. W., Guh, E., Yeh, J., Bhatt, S., Zhang, Y., et al. (2011). Development of single retinofugal axon arbors in normal and $\beta 2$ knock-out mice. J. Neurosci. 31, 3384-3399. doi: 10.1523/JNEUROSCI.4899-10.2011

Dunn, T. A., Storm, D. R., and Feller, M. B. (2009). Calcium-dependent increases in protein kinase-A activity in mouse retinal ganglion cells are mediated by multiple adenylate cyclases. PLoS One 4:e7877. doi: 10.1371/journal.pone. 0007877

Dunn, T. A., Wang, C.-T., Colicos, M. A., Zaccolo, M., DiPilato, L. M., Zhang, J., et al. (2006). Imaging of cAMP levels and protein kinase A activity reveals that retinal waves drive oscillations in second-messenger cascades. J. Neurosci. 26, 12807-12815. doi: 10.1523/jneurosci.3238-06.2006

Dyachok, O., Isakov, Y., Sågetorp, J., and Tengholm, A. (2006). Oscillations of cyclic AMP in hormone-stimulated insulin-secreting beta-cells. Nature 439, 349-352. doi: 10.1038/nature04410

Fagan, K. A., Graf, R. A., Tolman, S., Schaack, J., and Cooper, D. M. (2000). Regulation of a Ca2+-sensitive adenylyl cyclase in an excitable cell. Role of voltage-gated versus capacitative Ca2+ entry. J. Biol. Chem. 275, 40187-40194. doi: 10.1074/jbc.m006606200

Feldheim, D. A., Kim, Y. I., Bergemann, A. D., Frisén, J., Barbacid, M., and Flanagan, J. G. (2000). Genetic analysis of ephrin-A2 and ephrin-A5 shows their requirement in multiple aspects of retinocollicular mapping. Neuron 25, 563 574. doi: 10.1016/s0896-6273(00)81060-0

Feng, Q., Zhang, Y., Li, Y., Liu, Z., Zuo, J., and Fang, F. (2006). Two domains are critical for the nuclear localization of soluble adenylyl cyclase. Biochimie 88, 319328. doi: 10.1016/j.biochi.2005.09.003

Frisén, J., Yates, P. A., McLaughlin, T., Friedman, G. C., O'Leary, D. D., and Barbacid, M. (1998). Ephrin-A5 (AL-1/RAGS) is essential for proper retinal axon guidance and topographic mapping in the mammalian visual system. Neuron 20, 235-243. doi: 10.1016/s0896-6273(00)80452-3

Furman, M., Xu, H.-P., and Crair, M. C. (2013). Competition driven by retinal waves promotes morphological and functional synaptic development of neurons in the superior colliculus. J. Neurophysiol. 110, 1441-1454. doi: 10.1152/jn. 01066.2012

Gomez, T. M., Robles, E., Poo, M., and Spitzer, N. C. (2001). Filopodial calcium transients promote substrate-dependent growth cone turning. Science 291, 1983-1987. doi: 10.1126/science. 1056490

Gomez, T. M., Snow, D. M., and Letourneau, P. C. (1995). Characterization of spontaneous calcium transients in nerve growth cones and their effect on growth cone migration. Neuron 14, 1233-1246. doi: 10.1016/0896-6273(95) 90270-8

Gomez, T. M., and Spitzer, N. C. (1999). In vivo regulation of axon extension and pathfinding by growth-cone calcium transients. Nature 397, 350-355. doi: 10 . $1038 / 16927$

Gorbunova, Y. V., and Spitzer, N. C. (2002). Dynamic interactions of cyclic AMP transients and spontaneous Ca2+ spikes. Nature 418, 93-96. doi: 10. 1038/nature00835

Guirland, C., Suzuki, S., Kojima, M., Lu, B., and Zheng, J. Q. (2004). Lipid rafts mediate chemotropic guidance of nerve growth cones. Neuron 42, 51-62. doi: 10.1016/s0896-6273(04)00157-6

Hong, K., Nishiyama, M., Henley, J., Tessier-Lavigne, M., and Poo, M. (2000). Calcium signalling in the guidance of nerve growth by netrin-1. Nature 403 , 93-98. doi: 10.1038/47507

Hong, K. P., Spitzer, N. C., and Nicol, X. (2011). Improved molecular toolkit for cAMP studies in live cells. BMC Res. Notes 4:241. doi: 10.1186/1756-05004-241

Höpker, V. H., Shewan, D., Tessier-Lavigne, M., Poo, M., and Holt, C. (1999). Growth-cone attraction to netrin-1 is converted to repulsion by laminin-1. Nature 401, 69-73. doi: 10.1038/43441

Horvat, S. J., Deshpande, D. A., Yan, H., Panettieri, R. A., Codina, J., DuBose, T. D. Jr., et al. (2012). A-kinase anchoring proteins regulate compartmentalized cAMP signaling in airway smooth muscle. FASEB J. 26, 3670-3679. doi: 10.1096/fj.11201020

Imai, T., Suzuki, M., and Sakano, H. (2006). Odorant receptor-derived cAMP signals direct axonal targeting. Science 314, 657-661. doi: 10.1126/science. 1131794

Iwasato, T., Inan, M., Kanki, H., Erzurumlu, R. S., Itohara, S., and Crair, M. C. (2008). Cortical adenylyl cyclase 1 is required for thalamocortical synapse maturation and aspects of layer IV barrel development. J. Neurosci. 28, 59315943. doi: 10.1523/JNEUROSCI.0815-08.2008

Kleppisch, T. (2009). Phosphodiesterases in the central nervous system. Handb. Exp. Pharmacol. 191, 71-92. doi: 10.1007/978-3-540-68964-5_5

Kobayashi, T., Nagase, F., Hotta, K., and Oka, K. (2013). Crosstalk between second messengers predicts the motility of the growth cone. Sci. Rep. 3:3118. doi: 10 . 1038/srep03118

Landa, L. R. Jr., Harbeck, M., Kaihara, K., Chepurny, O., Kitiphongspattana, K., Graf, O., et al. (2005). Interplay of $\mathrm{Ca} 2+$ and cAMP signaling in the insulinsecreting MIN6 beta-cell line. J. Biol. Chem. 280, 31294-31302. doi: 10.1074/jbc. m505657200 
Li, L., Hutchins, B. I., and Kalil, K. (2009). Wnt5a induces simultaneous cortical axon outgrowth and repulsive axon guidance through distinct signaling mechanisms. J. Neurosci. 29, 5873-5883. doi: 10.1523/jneurosci.0183-09. 2009

Liu, S., Li, Y., Kim, S., Fu, Q., Parikh, D., Sridhar, B., et al. (2012). Phosphodiesterases coordinate cAMP propagation induced by two stimulatory G protein-coupled receptors in hearts. Proc. Natl. Acad. Sci. U S A 109, 65786583. doi: 10.1073/pnas.1117862109

Lu, H.-C., Butts, D. A., Kaeser, P. S., She, W.-C., Janz, R., and Crair, M. C. (2006). Role of efficient neurotransmitter release in barrel map development. J. Neurosci. 26, 2692-2703. doi: 10.1523/jneurosci.3956-05.2006

Luo, L., and Flanagan, J. G. (2007). Development of continuous and discrete neural maps. Neuron 56, 284-300. doi: 10.1016/j.neuron.2007.10.014

Luo, L., and O'Leary, D. D. M. (2005). Axon retraction and degeneration in development and disease. Annu. Rev. Neurosci. 28, 127-156. doi: 10.1146/annurev. neuro.28.061604.135632

Maritan, M., Monaco, G., Zamparo, I., Zaccolo, M., Pozzan, T., and Lodovichi, C. (2009). Odorant receptors at the growth cone are coupled to localized cAMP and Ca2+ increases. Proc. Natl. Acad. Sci. U S A 106, 3537-3542. doi: 10.1073/pnas. 0813224106

McConnachie, G., Langeberg, L. K., and Scott, J. D. (2006). AKAP signaling complexes: getting to the heart of the matter. Trends Mol. Med. 12, 317-323. doi: 10.1016/j.molmed.2006.05.008

Ming, G. L., Song, H. J., Berninger, B., Holt, C. E., Tessier-Lavigne, M., and Poo, M. M. (1997). cAMP-dependent growth cone guidance by netrin-1. Neuron 19, 1225-1235. doi: 10.1016/s0896-6273(00)80414-6

Moore, S. W., Lai Wing Sun, K., Xie, F., Barker, P. A., Conti, M., and Kennedy, T. E. (2008). Soluble adenylyl cyclase is not required for axon guidance to netrin-1. J. Neurosci. 28, 3920-3924. doi: 10.1523/jneurosci.0547-08.2008

Muir-Robinson, G., Hwang, B. J., and Feller, M. B. (2002). Retinogeniculate axons undergo eye-specific segregation in the absence of eye-specific layers. J. Neurosci. 22, 5259-5264.

Munck, S., Bedner, P., Bottaro, T., and Harz, H. (2004). Spatiotemporal properties of cytoplasmic cyclic AMP gradients can alter the turning behaviour of neuronal growth cones. Eur. J. Neurosci. 19, 791-797. doi: 10.1111/j.0953-816x.2004. 03118.x

Murray, A. J., Tucker, S. J., and Shewan, D. A. (2009). cAMP-dependent axon guidance is distinctly regulated by Epac and protein kinase A. J. Neurosci. 29, 15434-15444. doi: 10.1523/jneurosci.3071-09.2009

Nicol, X., Bennis, M., Ishikawa, Y., Chan, G. C.-K., Repérant, J., Storm, D. R., et al. (2006a). Role of the calcium modulated cyclases in the development of the retinal projections. Eur. J. Neurosci. 24, 3401-3414. doi: 10.1111/j.1460-9568. 2006.05227.x

Nicol, X., Hong, K. P., and Spitzer, N. C. (2011). Spatial and temporal second messenger codes for growth cone turning. Proc. Natl. Acad. Sci. U S A 108, 13776-13781. doi: 10.1073/pnas.1100247108

Nicol, X., Muzerelle, A., Rio, J. P., Métin, C., and Gaspar, P. (2006b). Requirement of adenylate cyclase 1 for the ephrin-A5-dependent retraction of exuberant retinal axons. J. Neurosci. 26, 862-872. doi: 10.1523/jneurosci.3385-05.2006

Nicol, X., Voyatzis, S., Muzerelle, A., Narboux-Nême, N., Südhof, T. C., Miles, R., et al. (2007). cAMP oscillations and retinal activity are permissive for ephrin signaling during the establishment of the retinotopic map. Nat. Neurosci. 10, 340-347. doi: 10.1038/nn1842

Nikolaev, V. O., Bünemann, M., Hein, L., Hannawacker, A., and Lohse, M. J. (2004). Novel single chain cAMP sensors for receptor-induced signal propagation. J. Biol. Chem. 279, 37215-37218. doi: 10.1074/jbc.C400302200

Nishiyama, M., Hoshino, A., Tsai, L., Henley, J. R., Goshima, Y., Tessier-Lavigne, M., et al. (2003). Cyclic AMP/GMP-dependent modulation of Ca2+ channels sets the polarity of nerve growth-cone turning. Nature 423, 990-995. doi: 10 . 1038/nature01751

Omori, K., and Kotera, J. (2007). Overview of PDEs and their regulation. Circ. Res. 100, 309-327. doi: 10.1161/01.res.0000256354.95791.f1

Ooashi, N., Futatsugi, A., Yoshihara, F., Mikoshiba, K., and Kamiguchi, H. (2005). Cell adhesion molecules regulate $\mathrm{Ca} 2+$-mediated steering of growth cones via cyclic AMP and ryanodine receptor type 3. J. Cell Biol. 170, 1159-1167. doi: 10. 1083/jcb.200503157

Penn, A. A., Riquelme, P. A., Feller, M. B., and Shatz, C. J. (1998). Competition in retinogeniculate patterning driven by spontaneous activity. Science 279, 2108 2112. doi: $10.1126 /$ science.279.5359.2108
Pham, T. A., Rubenstein, J. L., Silva, A. J., Storm, D. R., and Stryker, M. P. (2001). The CRE/CREB pathway is transiently expressed in thalamic circuit development and contributes to refinement of retinogeniculate axons. Neuron 31, 409-420. doi: 10.1016/s0896-6273(01)00381-6

Pietrobon, M., Zamparo, I., Maritan, M., Franchi, S. A., Pozzan, T., and Lodovichi, C. (2011). Interplay among cGMP, cAMP and Ca2+ in living olfactory sensory neurons in vitro and in vivo. J. Neurosci. 31, 8395-8405. doi: 10.1523/jneurosci. 6722-10.2011

Piggott, L. A., Bauman, A. L., Scott, J. D., and Dessauer, C. W. (2008). The Akinase anchoring protein Yotiao binds and regulates adenylyl cyclase in brain. Proc. Natl. Acad. Sci. U S A 105, 13835-13840. doi: 10.1073/pnas.0712100105

Polito, M., Klarenbeek, J., Jalink, K., Paupardin-Tritsch, D., Vincent, P., and Castro, L. R. V. (2013). The NO/cGMP pathway inhibits transient cAMP signals through the activation of PDE2 in striatal neurons. Front. Cell. Neurosci. 7:211. doi: 10. 3389/fncel.2013.00211

Ponsioen, B., Zhao, J., Riedl, J., Zwartkruis, F., van der Krogt, G., Zaccolo, M., et al. (2004). Detecting cAMP-induced Epac activation by fluorescence resonance energy transfer: Epac as a novel cAMP indicator. EMBO Rep. 5, 1176-1180. doi: 10.1038/sj.embor.7400290

Raslan, Z., and Naseem, K. M. (2014). Compartmentalisation of cAMP-dependent signalling in blood platelets: the role of lipid rafts and actin polymerisation. Platelets, 1-9. doi: 10.3109/09537104.2014.916792. [Epub ahead of print].

Ravary, A., Muzerelle, A., Hervé, D., Pascoli, V., Ba-Charvet, K. N., Girault, J.-A., et al. (2003). Adenylate cyclase 1 as a key actor in the refinement of retinal projection maps. J. Neurosci. 23, 2228-2238.

Robles, E., Huttenlocher, A., and Gomez, T. M. (2003). Filopodial calcium transients regulate growth cone motility and guidance through local activation of calpain. Neuron 38, 597-609. doi: 10.1016/s0896-6273(03)00260-5

Roisen, F. J., Murphy, R. A., Pichichero, M. E., and Braden, W. G. (1972). Cyclic adenosine monophosphate stimulation of axonal elongation. Science 175, 7374. doi: 10.1126/science.175.4017.73

Root, C. M., Velázquez-Ulloa, N. A., Monsalve, G. C., Minakova, E., and Spitzer, N. C. (2008). Embryonically expressed GABA and glutamate drive electrical activity regulating neurotransmitter specification. J. Neurosci. 28, 4777-4784. doi: 10.1523/jneurosci.4873-07.2008

Rossi, F. M., Pizzorusso, T., Porciatti, V., Marubio, L. M., Maffei, L., and Changeux, J.-P. (2001). Requirement of the nicotinic acetylcholine receptor $\beta 2$ subunit for the anatomical and functional development of the visual system. Proc. Natl. Acad. Sci. U S A 98, 6453-6458. doi: 10.1073/pnas.101120998

Ryu, M.-H., Moskvin, O. V., Siltberg-Liberles, J., and Gomelsky, M. (2010). Natural and engineered photoactivated nucleotidyl cyclases for optogenetic applications. J. Biol. Chem. 285, 41501-41508. doi: 10.1074/jbc.M110.177600

Sample, V., DiPilato, L. M., Yang, J. H., Ni, Q., Saucerman, J. J., and Zhang, J. (2012). Regulation of nuclear PKA revealed by spatiotemporal manipulation of cyclic AMP. Nat. Chem. Biol. 8, 375-382. doi: 10.1038/nchembio.799

Schröder-Lang, S., Schwärzel, M., Seifert, R., Strünker, T., Kateriya, S., Looser, J., et al. (2007). Fast manipulation of cellular cAMP level by light in vivo. Nat. Methods 4, 39-42. doi: 10.1038/nmeth975

Serizawa, S., Miyamichi, K., Takeuchi, H., Yamagishi, Y., Suzuki, M., and Sakano, H. (2006). A neuronal identity code for the odorant receptor-specific and activitydependent axon sorting. Cell 127, 1057-1069. doi: 10.1016/j.cell.2006.10.031

Shelly, M., Cancedda, L., Heilshorn, S., Sumbre, G., and Poo, M.-M. (2007). LKB1/STRAD promotes axon initiation during neuronal polarization. Cell 129, 565-577. doi: 10.1016/j.cell.2007.04.012

Shelly, M., Cancedda, L., Lim, B. K., Popescu, A. T., Cheng, P., Gao, H., et al. (2011). Semaphorin3A regulates neuronal polarization by suppressing axon formation and promoting dendrite growth. Neuron 71, 433-446. doi: 10.1016/j.neuron. 2011.06.041

Shelly, M., Lim, B. K., Cancedda, L., Heilshorn, S. C., Gao, H., and Poo, M. (2010). Local and long-range reciprocal regulation of cAMP and cGMP in axon/dendrite formation. Science 327, 547-552. doi: 10.1126/science.1179735

Shewan, D., Dwivedy, A., Anderson, R., and Holt, C. E. (2002). Age-related changes underlie switch in netrin-1 responsiveness as growth cones advance along visual pathway. Nat. Neurosci. 5, 955-962. doi: 10.1038/nn919

Song, H. J., Ming, G. L., and Poo, M. M. (1997). cAMP-induced switching in turning direction of nerve growth cones. Nature 388, 275-279. doi: 10.1038/40864

Stellwagen, D., and Shatz, C. J. (2002). An instructive role for retinal waves in the development of retinogeniculate connectivity. Neuron 33, 357-367. doi: 10. 1016/s0896-6273(02)00577-9 
Stellwagen, D., Shatz, C. J., and Feller, M. B. (1999). Dynamics of retinal waves are controlled by cyclic AMP. Neuron 24, 673-685. doi: 10.1016/s08966273(00)81121-6

Stierl, M., Stumpf, P., Udwari, D., Gueta, R., Hagedorn, R., Losi, A., et al. (2011). Light modulation of cellular cAMP by a small bacterial photoactivated adenylyl cyclase, bPAC, of the soil bacterium Beggiatoa. J. Biol. Chem. 286, 1181-1188. doi: 10.1074/jbc.M110.185496

Tang, F., Dent, E. W., and Kalil, K. (2003). Spontaneous calcium transients in developing cortical neurons regulate axon outgrowth. J. Neurosci. 23, 927-936.

Terrin, A., Di Benedetto, G., Pertegato, V., Cheung, Y.-F., Baillie, G., Lynch, M. J., et al. (2006). PGE(1) stimulation of HEK293 cells generates multiple contiguous domains with different [cAMP]: role of compartmentalized phosphodiesterases. J. Cell Biol. 175, 441-451. doi: 10.1083/jcb.200605050

Terrin, A., Monterisi, S., Stangherlin, A., Zoccarato, A., Koschinski, A., Surdo, N. C., et al. (2012). PKA and PDE4D3 anchoring to AKAP9 provides distinct regulation of cAMP signals at the centrosome. J. Cell Biol. 198, 607-621. doi: 10. 1083/jcb.201201059

Tojima, T., Itofusa, R., and Kamiguchi, H. (2009). The nitric oxide-cGMP pathway controls the directional polarity of growth cone guidance via modulating cytosolic Ca2+ signals. J. Neurosci. 29, 7886-7897. doi: 10.1523/jneurosci.008709.2009

Valsecchi, F., Konrad, C., and Manfredi, G. (2014). Role of soluble adenylyl cyclase in mitochondria. Biochim. Biophys. Acta doi: 10.1016/j.bbadis.2014.05. 035. [Epub ahead of print].

Vincent, P., Castro, L. R. V., Gervasi, N., Guiot, E., Brito, M., and PaupardinTritsch, D. (2012). PDE4 control on cAMP/PKA compartmentation revealed by biosensor imaging in neurons. Horm. Metab. Res. 44, 786-789. doi: 10.1055/s0032-1311631

Welker, E., Armstrong-James, M., Bronchti, G., Ourednik, W., GheorghitaBaechler, F., Dubois, R., et al. (1996). Altered sensory processing in the somatosensory cortex of the mouse mutant barrelless. Science 271, 1864-1867. doi: $10.1126 /$ science.271.5257.1864

Willoughby, D., and Cooper, D. M. F. (2006). Ca2+ stimulation of adenylyl cyclase generates dynamic oscillations in cyclic AMP. J. Cell Sci. 119, 828-836. doi: 10 $1242 /$ jcs. 02812

Willoughby, D., and Cooper, D. M. F. (2007). Organization and Ca2+ regulation of adenylyl cyclases in cAMP microdomains. Physiol. Rev. 87, 965-1010. doi: 10. 1152/physrev.00049.2006

Willoughby, D., Everett, K. L., Halls, M. L., Pacheco, J., Skroblin, P., Vaca, L., et al. (2012). Direct binding between Orail and AC8 mediates dynamic interplay between Ca2+ and cAMP signaling. Sci. Signal. 5:ra29. doi: 10.1126/scisignal. 2002299

Willoughby, D., Masada, N., Wachten, S., Pagano, M., Halls, M. L., Everett, K. L., et al. (2010). AKAP79/150 interacts with AC8 and regulates Ca2+-dependent
cAMP synthesis in pancreatic and neuronal systems. J. Biol. Chem. 285, 2032820342. doi: 10.1074/jbc.m110.120725

Wong, W., and Scott, J. D. (2004). AKAP signalling complexes: focal points in space and time. Nat. Rev. Mol. Cell Biol. 5, 959-970. doi: 10.1038/nrm1527

Wong, S. T., Trinh, K., Hacker, B., Chan, G. C., Lowe, G., Gaggar, A., et al. (2000). Disruption of the type III adenylyl cyclase gene leads to peripheral and behavioral anosmia in transgenic mice. Neuron 27, 487-497. doi: 10.1016/s08966273(00)00060-X

Wu, K. Y., Zippin, J. H., Huron, D. R., Kamenetsky, M., Hengst, U., Buck, J., et al. (2006). Soluble adenylyl cyclase is required for netrin-1 signaling in nerve growth cones. Nat. Neurosci. 9, 1257-1264. doi: 10.1038/nn1767

Zaccolo, M., and Movsesian, M. A. (2007). cAMP and cGMP signaling cross-talk: role of phosphodiesterases and implications for cardiac pathophysiology. Circ. Res. 100, 1569-1578. doi: 10.1161/circresaha.106.144501

Zhang, J., Ackman, J. B., Xu, H.-P., and Crair, M. C. (2012). Visual map development depends on the temporal pattern of binocular activity in mice. Nat. Neurosci. 15, 298-307. doi: 10.1038/nn.3007

Zhang, J., Ma, Y., Taylor, S. S., and Tsien, R. Y. (2001). Genetically encoded reporters of protein kinase A activity reveal impact of substrate tethering. Proc. Natl. Acad. Sci. U S A 98, 14997-15002. doi: 10.1073/pnas.211566798

Zippin, J. H., Chen, Y., Nahirney, P., Kamenetsky, M., Wuttke, M. S., Fischman, D. A., et al. (2003). Compartmentalization of bicarbonate-sensitive adenylyl cyclase in distinct signaling microdomains. FASEB J. 17, 82-84. doi: 10.1096/fj. 02-0598fje

Zou, D.-J., Chesler, A. T., Le Pichon, C. E., Kuznetsov, A., Pei, X., Hwang, E. L., et al. (2007). Absence of adenylyl cyclase 3 perturbs peripheral olfactory projections in mice. J. Neurosci. 27, 6675-6683. doi: 10.1523/jneurosci.0699-07.2007

Conflict of Interest Statement: The authors declare that the research was conducted in the absence of any commercial or financial relationships that could be construed as a potential conflict of interest.

Received: 10 September 2014; paper pending published: 05 October 2014; accepted: 21 October 2014; published online: 13 November 2014.

Citation: Averaimo $S$ and Nicol X (2014) Intermingled cAMP, cGMP and calcium spatiotemporal dynamics in developing neuronal circuits. Front. Cell. Neurosci. 8:376. doi: $10.3389 /$ fncel.2014.00376

This article was submitted to the journal Frontiers in Cellular Neuroscience.

Copyright (C) 2014 Averaimo and Nicol. This is an open-access article distributed under the terms of the Creative Commons Attribution License (CC BY). The use, distribution and reproduction in other forums is permitted, provided the original author(s) or licensor are credited and that the original publication in this journal is cited, in accordance with accepted academic practice. No use, distribution or reproduction is permitted which does not comply with these terms. 